\title{
Three-color reconfigurable organic light-emitting devices
}

\author{
Chung-Chih Wu, ${ }^{\text {a) }}$ Chieh-Wei Chen, and Ting-Yi Cho \\ Department of Electrical Engineering, Graduate Institute of Electro-optical Engineering and Graduate \\ Institute of Electronics Engineering, National Taiwan University, Taipei, Taiwan 10617, Republic of China
}

(Received 2 January 2003; accepted 14 May 2003)

\begin{abstract}
This report reveals utilization of phase transitions and corresponding changes in physical properties of organic semiconductors for implementing reconfigurable organic optoelectronic devices, i.e., a device whose configurations and characteristics can be programed after fabrication. Specifically, glass transitions of amorphous molecular materials have been exploited to demonstrate reconfigurable organic light-emitting devices (OLEDs) capable of generating any of the three primary colors. The capability to pattern such devices into fine color pixels with thermal imaging renders it attractive for applications in high-resolution full-color OLED displays and as active optical memory devices. (C) 2003 American Institute of Physics. [DOI: 10.1063/1.1595724]
\end{abstract}

In many aspects organic molecular semiconductors are reproducing operations of various electronic and optoelectronic devices that are conventionally made from inorganic semiconductors. ${ }^{1-4}$ Some truly unique features belonging only to organic materials, however, have seldom been explored for active devices. Among these is the "reconfigurable" nature of molecular materials. For instance, within accessible ranges of temperature, many molecular solids undergo phase transitions, each of which induces substantial changes in arrangement of molecules, morphology, and corresponding physical properties. When appropriately manipulated, these phenomena may be employed to construct reconfigurable optoelectronic devices having additional functionality. Here, utilizing the glass transitions of amorphous molecular materials, we demonstrate a three-color reconfigurable organic light-emitting device (OLED), whose device configuration can be determined after fabrication and emission color can be altered correspondingly from blue to green and to red. Moreover, our work establishes the fine patterning of color pixels through thermal imaging of such reconfigurable devices.

Amorphous molecular materials, i.e., molecular glasses, are low-molar-mass organic compounds with a stable amorphous phase above room temperature. ${ }^{5}$ Such materials have found wide applications in recent years in organic optoelectronics, such as photoconductors, OLEDs, photovoltaics, etc., due to their capability to form uniform thin films. ${ }^{5-8} \mathrm{An}$ organic glass is usually characterized by its glass transition temperature $\left(T_{g}\right)$. Below $T_{g}$, the condensed phase can be regarded as an amorphous solid. Across $T_{g}$, there occurs no major morphological change, yet the viscosity is substantially reduced and freedom of molecular motion becomes larger, rendering the condensed phase more like a supercooled liquid. ${ }^{5}$ Consequently, diffusion and interdiffusion that could hardly occur below $T_{g}$ for a molecular glass are usually substantially enhanced. ${ }^{9,10}$ Indeed, this has long been the principle behind an electronic printing process, dyediffusion thermal transfer printing. ${ }^{11}$

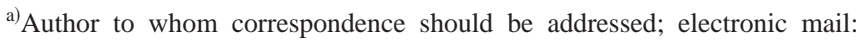
chungwu@cc.ee.ntu.edu.tw
}

Such property could be used in a multilayer organic device to "fuse" initially separated functional layers by inserting a sacrificial fusing layer with a lower $T_{g}$, giving a feasible way to form graded organic junctions or to alter ("program") device characteristics. ${ }^{12,13}$ We have thus previously demonstrated a reconfigurable two-color OLED that emits blue light as fabricated but can be transformed into a green-emitting one. ${ }^{12}$ Since only one type of device needs to be fabricated and no other schemes of color extraction are required, in comparison with existing approaches, ${ }^{14}$ such reconfigurable devices may be a simpler alternative to color patterning in OLED applications. The programability of reconfigurable OLEDs may also render applications possible, such as active optical memory devices. In practice, however, requirements and challenges remain in development of general device architectures that can be reconfigured to emit any of the three primary colors and in fine patterning of such devices into color pixels. Here we describe the proof of principle of a three-color reconfigurable OLED and its fine patterning, making use of unique phase transitions of different organic materials.

The schematic energy level diagram for one possible structure of the reconfigurable three-color device is shown in Fig. 1(a). The as-deposited structure on the glass substrate has the typical structure of multiple organic layers sandwiched between the bottom indium-tin-oxide (ITO) anode and the top metal cathode. The stack of organic layers in sequence consists of a hole-injection layer (HIL), a holetransport and blue emitting layer (HTL/BEL), a first holeblocking and electron-transport layer (HBL/ETL-1) with a glass transition temperature of $T_{g 1}$, an electron-transport and green emitting layer (ETL/GEL), a second hole-blocking and electron-transport layer (HBL/ETL-2) with a glass transition temperature $T_{g_{2}}$, and finally an electron-transport and red emitting layer (ETL/REL). HBL/ETL-1 and HBL/ETL-2 are chosen to have lower $T_{g}$ 's than remaining layers and $T_{g 1}$ $<T_{g 2}$.

In the as-fabricated configuration, one would expect that under forward bias injected holes be confined in the HTL/BEL while injected electrons transport all the way to recombine with holes, generating blue emission from 

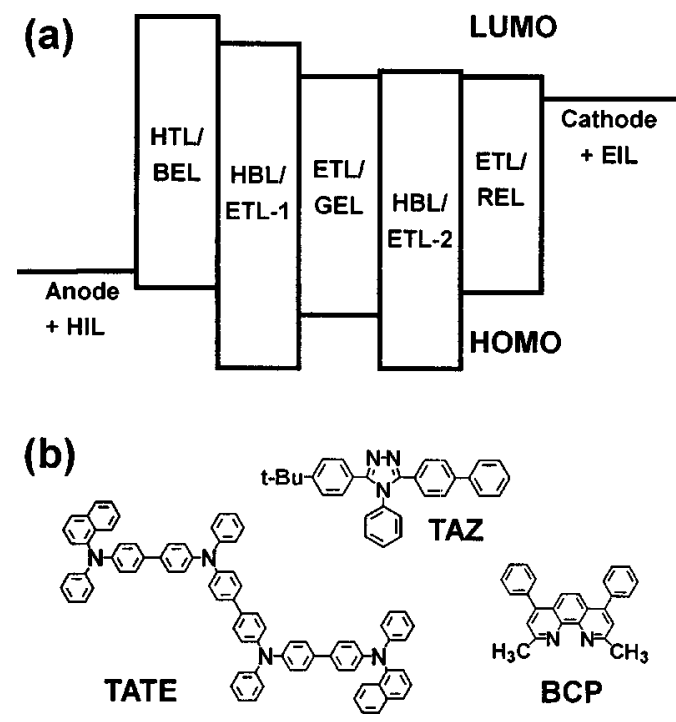

FIG. 1. (a) Schematic energy level diagram of the device, and (b) chemical structures of some materials used. HOMO and LUMO represent highest occupied molecular orbital and lowest unoccupied molecular orbital, respectively.

HTL/BEL. To transform the device into a green-emitting one, one may raise the device temperature over $T_{g 1}$ to induce interdiffusion through HBL/ETL-1 and to bridge (mix) HTL/ BEL and ETL/GEL. As a consequence, the hole-blocking property of HBL/ETL-1 may be bypassed, permitting holes to reach ETL/GEL. As still blocked by HBL-ETL-2, these holes would recombine with electrons injected into ETL/ GEL, resulting in green emission. Further, one may again transform the device into a red emitting one by raising the device temperature over $T_{g 2}$ to induce interdiffusion through HBL/ETL-2, bridging materials and bypassing the blocking property again. With the HBL/ETL-1, ETL/GEL and HBL/ ETL-2 thin enough, direct bridging of HTL/BEL and ETL/ REL might occur such that holes could reach ETL/REL or its neighborhood to recombine with electrons around. As such, one might expect emission from the material with the smallest energy gap, i.e., red emission, through either direct recombination on or resonant energy transfer to ETL/REL molecules. In summary, the present architecture of reconfigurable devices in general employs fusable carrier-blocking layers inserted between carrier-transport/emitting layers to function as switchable gates for regulating the flow and distribution of carriers.

Actual implementation of the earlier device has been carried out using the following materials [Fig. 1(b)]: LiF (5 $\AA) / \mathrm{Al}$ as the bilayer cathode, ${ }^{15}$ conducting polymer polyethylene dioxythiophene/polystyrene sulphonate (PEDT:PSS) (Bayer Corp., $300 \AA$ ) as HIL, ${ }^{16} N, N^{\prime}$-diphenyl- $N, N^{\prime}$-bis $\left(4^{\prime}\right.$ [N,N-bis(naphth-1-yl)-amino]-biphenyl-4-yl)-benzidine (a triarylamine tetramer (TATE) $T_{g} \sim 150{ }^{\circ} \mathrm{C}, 400 \AA$ ) as HTL/BEL, ${ }^{17}$ 3-(4'-tert-butylphenyl)-4-phenyl-5-(4"-biphenyl)-1,2,4-triazole (TAZ) $\quad\left(T_{g} \sim 70{ }^{\circ} \mathrm{C}, \quad 75 \AA \begin{array}{l}\mathrm{A} \\ \text { ) as }\end{array}\right.$ HBL/ETL- $1,{ }^{18}$ tris-(8-hydroxyquinoline) aluminum $\left(\mathrm{Alq}_{3}\right)$ $\left(T_{g} \sim 170^{\circ} \mathrm{C}, \quad 75 \AA\right)$ as $\mathrm{ETL} / \mathrm{GEL},{ }^{1} \quad$ 2,9-dimethyl-4,7diphenyl-1,10-phenanthroline (BCP) $\left(T_{g} \sim 85^{\circ} \mathrm{C}, 100 \AA\right)$ as HBL/ETL-2, ${ }^{19} 150 \AA \mathrm{Alq}_{3}$ doped with red dye DCJTB (2 wt. \%) plus $350 \AA$ pure $\mathrm{Alq}_{3}$ as ETL/REL. ${ }^{20}$ PEDT:PSS was prepared by spin coating and all other layers were deposited
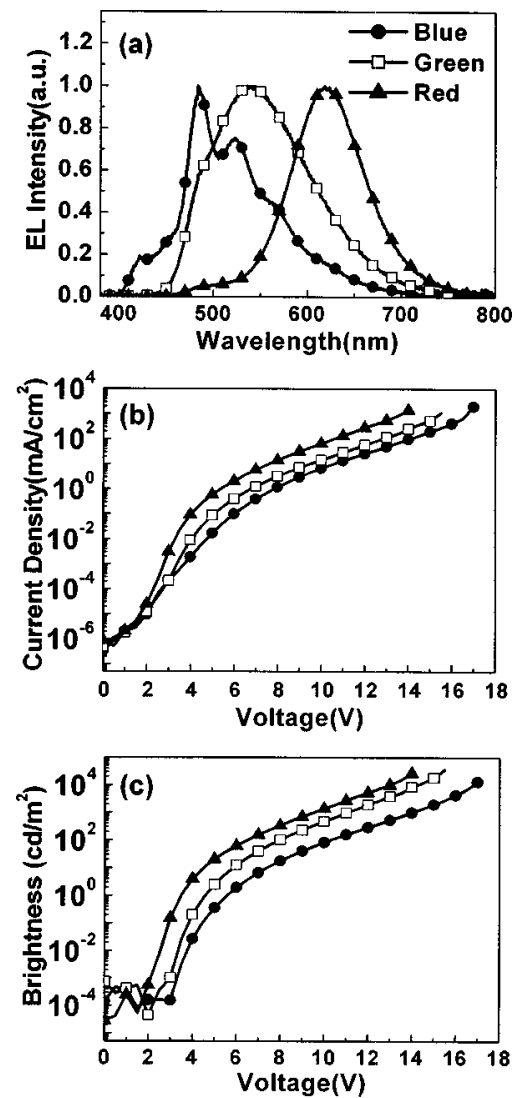

FIG. 2. (a) Electroluminescence spectra, (b) $I-V$, and (c) $L-V$ characteristics of an as-fabricated blue device, a reconfigured green device and a reconfigured red device.

by vacuum deposition in a multiple-source vacuum chamber within one vacuum pump-down. $T_{g}$ 's of materials were determined by differential scanning calorimetry. In performing interdiffusion, it was found that a temperature of $\sim 95^{\circ} \mathrm{C}$ is enough for inducing effective interdiffusion through TAZ, while $\sim 125^{\circ} \mathrm{C}$ is necessary for BCP. To endure these relatively high transformation temperatures, high- $T_{g}$ TATE was chosen as the HTL/BEL.

Figure 2 compares the spectral and the current-voltagebrightness $(I-V-L)$ characteristics of a device in the asfabricated blue operation, in the reconfigured green operation and in the reconfigured red operation. The transformation from blue to green was performed by annealing at $\sim 95^{\circ} \mathrm{C}$ for $3 \mathrm{~min}$, and the transformation from green to red by another annealing at $\sim 125{ }^{\circ} \mathrm{C}$ for $3 \mathrm{~min}$. The electroluminescence spectra in the blue, green and red operations [Fig. 2(a)] show emission dominantly from TATE, $\mathrm{Alq}_{3}$, and DCJTB, respectively, confirming the proposed device operation. Devices in all different operations show typical $I-V-L$ characteristics of a well behaved OLED, with a low turn-on voltage of $2-3 \mathrm{~V}$ (defined as the voltage where emission becomes detectable). In the $I-V$ characteristics, there is a consistent shift to lower voltage with the transformation from blue to green and to red operation. This is consistent with previous reports that replacement of the abrupt junction with a mixed layer or a graded junction usually leads to reduction of device voltage. ${ }^{12,13,21,22}$

The devices in all operations endure current densities up to $800-1200 \mathrm{~mA} / \mathrm{cm}^{2}$. Correspondingly, devices in the blue,
to AIP license or copyright, see http://ojps.aip.org/aplo/aplcr.jsp 
(a)

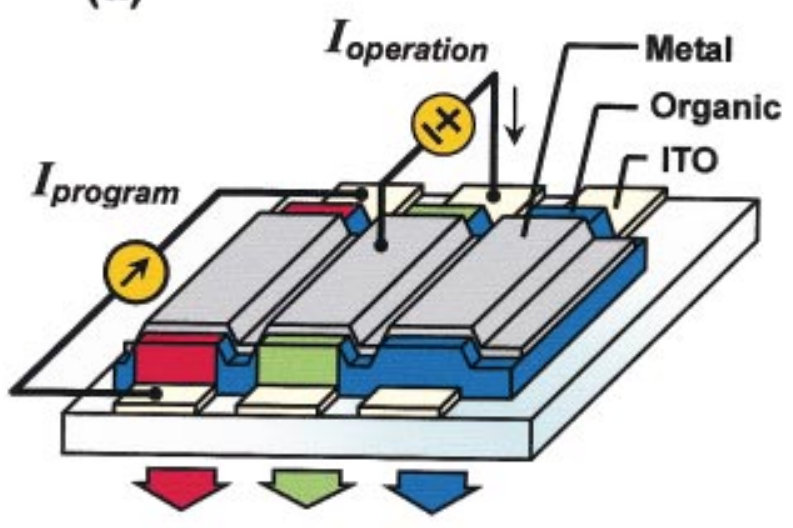

(b)

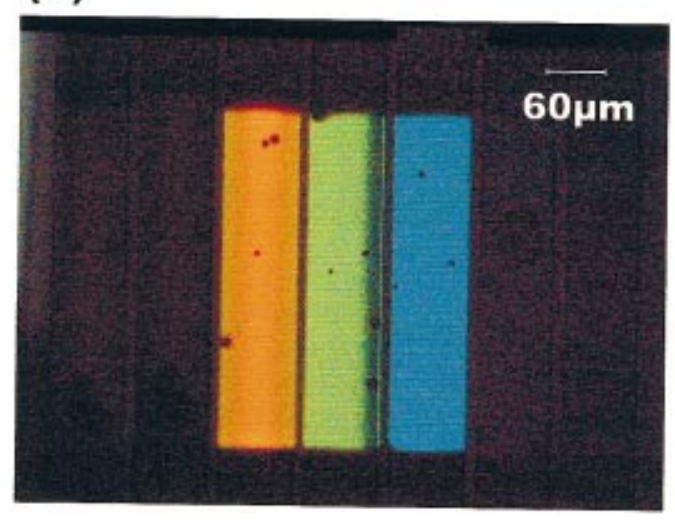

FIG. 3. (Color) Fine patterning of color pixels using reconfigurable OLEDs. (a) A schematic diagram showing the structure and how patterning is performed. (b) Photograph of the patterned blue, green and red devices operated at current densities of 22,14 , and $18 \mathrm{~mA} / \mathrm{cm}^{2}$, respectively. The sheet resistance of the ITO lines is $15 \Omega / \square$. The programing currents for green and red are $(17 \mathrm{~mA} / 80 \mu \mathrm{m}, 12 \mathrm{~s})$ and $(21.5 \mathrm{~mA} / 80 \mu \mathrm{m}, 4 \mathrm{~s})$, respectively, reaching device internal temperatures of $\sim 110^{\circ} \mathrm{C}$ (green) and $\sim 130^{\circ} \mathrm{C}$ (red) as detected by an infrared thermal imaging camera NEC thermal tracer.

green, and red operations exhibit brightness's up to 10000 , 40000 , and $30000 \mathrm{~cd} / \mathrm{m}^{2}$ and peak quantum efficiencies of $\sim 1 \%, 1.2 \%$, and $3.1 \%$ photon/electron, respectively. The lower efficiencies of blue and green emission are primarily limited by the luminescence quantum yields of emitting materials currently used. These devices indeed exhibit substantial improvement in the device operation range in comparison with the reconfigurable two-color device in the previous work, ${ }^{12}$ where a HBL/ETL of very low $T_{g}\left(\sim 30^{\circ} \mathrm{C}\right)$ was used and therefore the pristine (blue) operation could remain only up to $\sim 200 \mathrm{~mA} / \mathrm{cm}^{2}$ and $1000 \mathrm{~cd} / \mathrm{m}^{2}$ before transformation. Furthermore, the present devices in the pristine configuration are stable up to $60-70^{\circ} \mathrm{C}$ under extended annealing $(>100 \mathrm{~h})$, in contrast with $\sim 30^{\circ} \mathrm{C}$ in previous devices. These improvements are attributed mainly to higher- $T_{g}$ HBL/ETLs used in the present work. Indeed, it had become difficult to perform transformation by directly running large programing current pulses through the device without causing catastrophic destruction, as was done in the previous work. Nevertheless, the reconfiguration and fine patterning of color pixels could be realized instead by selective external heating, using thermal imaging techniques developed for conventional thermal printing. ${ }^{11}$ Figure 3(a) illustrates one such scheme for fine patterning, in which ITO anode lines are driven by high programing current pulses to induce resistive heating. By running different programing currents, devices on a line can be selectively transformed to green or red ones. Figure 3(b) shows the photograph of the thus produced fine color pixels (with a line width and spacing of 80 and $12 \mu \mathrm{m}$, respectively) under electrical excitation. The programing currents required typically are nearly two-order higher than the maximal endurable operation currents of devices, rendering a low possibility of accidental programing during device operation.

In summary, glass transitions of amorphous molecular materials have been exploited to demonstrate reconfigurable OLEDs capable of generating any of the three primary colors. The capability to fine pattern such device with thermal imaging renders it attractive for use in making highresolution full-color OLED displays and as active optical memory devices.

The authors would like to acknowledge financial support from National Science Council and Ministry of Education of the Republic of China.

${ }^{1}$ C. W. Tang and S. A. VanSlyke, Appl. Phys. Lett. 51, 913 (1987).

${ }^{2}$ C. W. Tang, Appl. Phys. Lett. 48, 183 (1986).

${ }^{3}$ F. Garnier, G. Horowitz, X. Peng, and D. Fichou, Adv. Mater. (Weinheim, Ger.) 2, 592 (1990).

${ }^{4}$ P. Peumans, V. Bulović, and S. R. Forrest, Appl. Phys. Lett. 76, 3855 (2000).

${ }^{5}$ K. Naito and A. Miura, J. Phys. Chem. 97, 6240 (1993).

${ }^{6}$ Y. Shirota, J. Mater. Chem. 10, 1 (2000).

${ }^{7}$ P. Strohriegl and J. V. Grazulevicius, Adv. Mater. (Weinheim, Ger.) 14, 1439 (2002)

${ }^{8}$ P. M. Borsenberger and D. S. Weiss, Organic Photoreceptors for Imaging Systems (Marcel Dekker, New York, 1993).

${ }^{9}$ D. Ehlich and H. Sillescu, Macromolecules 26, 1600 (1990).

${ }^{10}$ P. F. Nealey, R. E. Cohen, and A. S. Argon, Macromolecules 26, 1287 (1993).

${ }^{11}$ P. Gregory, Chemistry and Technology of Printing and Imaging Systems (Blackie Academic \& Professional, Bishopbriggs, 1996).

${ }^{12}$ C.-C. Wu, C.-W. Chen, Y.-T. Lin, H.-L. Yu, J.-H. Hsu, and T.-Y. Luh, Appl. Phys. Lett. 79, 3023 (2001).

${ }^{13}$ C.-W. Chen, T.-Y. Cho, C.-C. Wu, H.-L. Yu, and T.-Y. Luh, Appl. Phys. Lett. 81, 1570 (2002).

${ }^{14}$ P. E. Burrows, G. Gu, V. Bulović, Z. Shen, S. R. Forrest, and M. E. Thompson, IEEE Trans. Electron Devices 44, 1188 (1997).

${ }^{15}$ L. S. Hung, C. W. Tang, and M. G. Mason, Appl. Phys. Lett. 70, 152 (1997).

${ }^{16}$ A. Elschner, F. Bruder, H. W. Heuer, F. Jonas, A. Karbach, S. Kirchmeyer, S. Thurm, and R. Wehrmann, Synth. Met. 111, 139 (2000).

${ }^{17}$ S. Tokito, H. Tanaka, K. Noda, A. Okada, and Y. Taga, Appl. Phys. Lett. 70, 1929 (1997).

${ }^{18}$ J. Kido, K. Hongawa, K. Okuyama, and K. Nagai, Appl. Phys. Lett. 63, 2627 (1993).

${ }^{19}$ Y. Kijima, N. Asai, and S. Tamura, Jpn. J. Appl. Phys., Part 1 38, 5274 (1999).

${ }^{20}$ C. H. Chen, J. Shi, and C. W. Tang, Macromol. Symp. 125, 1 (1997).

${ }^{21}$ A. B. Chwang, R. C. Kwong, and J. J. Brown, Appl. Phys. Lett. 80, 725 (2002).

${ }^{22}$ H. Riel, W. Brütting, T. Beierlein, E. Haskal, P. Müller, and W. Riess, Synth. Met. 111-112, 303 (2000). 